\title{
Novel combined shield design for eye and face protection from COVID-19
}

\author{
Xiu-Ling Huang ${ }^{1} \cdot$ Jin-Rong Yang ${ }^{1} \cdot$ Yu-Xiang Sun ${ }^{1} \cdot$ Yi-Wen Chen ${ }^{1}$. \\ Xiu-Mei Wang ${ }^{1} \cdot$ Shui-Miao Du ${ }^{2} \cdot$ Zi-Kai Hua $^{1}$
}

Received: 30 July 2020/Revised: 24 September 2020/Accepted: 24 November 2020/Published online: 6 January 2021

(C) Shanghai University and Springer-Verlag GmbH Germany, part of Springer Nature 2021

\begin{abstract}
The World Health Organization emphasized the importance of goggles and face shields for protection of medical personnel at the outbreak of the COVID-19 pandemic. Unsurprisingly, almost all countries suffered from a critical supply shortage of goggles and face shields, as well as many other types of personal protective equipment (PPE), for a long period, owing to the lack of key medical material supplies and the inefficiency of existing fabrication methods arising from the need to avoid crowds during the outbreak of COVID-19. In this paper, we propose a novel combined shield design for eye and face protection that can be rapidly fabricated using three-dimensional printing technology. The designed prototype eye-face shield is accessible to the general public, offering more possibilities for yield improvement in PPE during emergent infectious disease events such as COVID-19.
\end{abstract}

Keywords Goggles · Face shield · Three-dimensional (3D) printing $\cdot$ COVID-19

\section{Introduction}

Since March 2020, the coronavirus disease (COVID-19) has become a worldwide pandemic. COVID-19 is transmitted via droplets and fomites during close unprotected

Zi-Kai Hua

eddie_hua@shu.edu.cn

1 School of Mechatronic Engineering and Automation, Shanghai University, Shanghai 200444, People's Republic of China

2 Sino European School of Technology of Shanghai University, Shanghai 200444, People's Republic of China contact, and transmission through the ocular surface should not be ignored $[1,2]$. According to China's anti-epidemic experience, personal protective equipment (PPE) for doctors includes masks (N95/disposable medical masks being the most effective), protective clothing, disposable gloves, goggles, and face shields [3, 4]. The protection of the eyes and face is very important. When a face shield is used in conjunction with a mask and goggles, the former immediately keeps the vast majority of the potentially infectious droplets at a reasonable physical distance, while the latter offers locally enhanced protection in a further step. However, it is both inconvenient and uncomfortable for medical staff to wear traditional face shields and goggles simultaneously (particularly for long periods), owing to the extra weight of the frame of the face shield. Therefore, we propose a combined design of goggles and shields based on the three-dimensional (3D) rapid manufacturing mode. We designed a special structure to quickly and easily assemble the goggles and face shields while removing the frame required by traditional face shields. 3D printing facilitates the production of PPEs with a shortage of materials during the COVID-19 pandemic.

Any company or individual with 3D printing equipment can freely obtain our eye-face shield model files through the link provided at the end of the article for production. Similarly, Tino [5] and Neijhoft [6] used a 3D-printing method to make protective equipment such as masks and face shields and provided an open-access model that could be downloaded. Our design has been used in hospitals in China since February 5th 2020. To our knowledge, no medical staff who used our eye-face shields were reported to be infected with COVID-19. Therefore, the clinical effectiveness of the eye-face shield has been preliminarily confirmed. The main objective of this study was to provide a novel design and fabrication method for the rapid 
production of all-in-one eye-face shields. Our design can serve as either an alternative or a complement to traditional fabrication methods of goggles and face shields, according to the practical requirements.

\section{Design of eye-face shield}

The eye-face shield is composed of four parts: goggles, lenses, face shields, and elastic bands. The goggles protect the eyes and support the face shield with a special connection structure. The requirements for goggles used in clinical settings are presented in Table 1.

We designed goggles with three different sizes (dimensions are shown in Table 2). The size was based on the Chinese GB 14866-2006 standard, which mainly refers to ISO 4849:1981, as well as the goggles on the market. According to the requirements and dimensions of the aforementioned goggles, we created a 3D model of the goggles can be opened and modified by SolidWorks, NX, 3Dmax, and other 3D modeling software programs. We found defects through sample try-on and repeatedly modified the model to improve the airtightness, comfort, and aesthetics.

For satisfying requirement $\mathrm{A}$ in Table 1, the goggles do not have any holes except for the lens holes (see Fig. 1) and the elastic-band holes (see " 3 " in Fig. 1). We adjusted the arc of the goggles to fit the face and improve the airtightness. We designed an inverted "V" shape at the nose wings (see Fig. 1) to fit the slope of the nose bridge.

For satisfying requirement $\mathrm{B}$ in Table 1, the edges that fit the face are designed with flanging. The outward flanging (see " 4 " in Fig. 1) is $12 \mathrm{~mm}$ wide and approximately $90^{\circ}$ from the horizontal plane. The inward flanging at the nose (see " 5 " in Fig. 1) has a width of $5 \mathrm{~mm}$. The flanging improves the airtightness, increases the stress area, and reduces the number of pressure sores.

For satisfying requirement $\mathrm{C}$ in Table 1 , we designed anchors on both sides of the goggles (see "7" in Fig. 1) to connect the face shield. There are four anchors: two on the left and two on the right.

Table 1 Properties of a nice goggle

\begin{tabular}{ll}
\hline Requirement & Properties \\
\hline A & Excellent airtightness \\
B & Ultimate comfort for long time wearing \\
C & Ease of assembling/disassembling \\
D & Ease of lenses fabricating \\
E & Aesthetic appearance and tomographical smoothness \\
\hline
\end{tabular}

Table 2 Dimensions of the three size goggle face

\begin{tabular}{ll}
\hline Size & Dimensions of goggle face \\
\hline Large size & Length $\times$ width $=164 \mathrm{~mm} \times 70 \mathrm{~mm}$ \\
Medium size & Length $\times$ width $=150 \mathrm{~mm} \times 70 \mathrm{~mm}$ \\
Small size & Length $\times$ width $=110 \mathrm{~mm} \times 50 \mathrm{~mm}$ \\
\hline
\end{tabular}

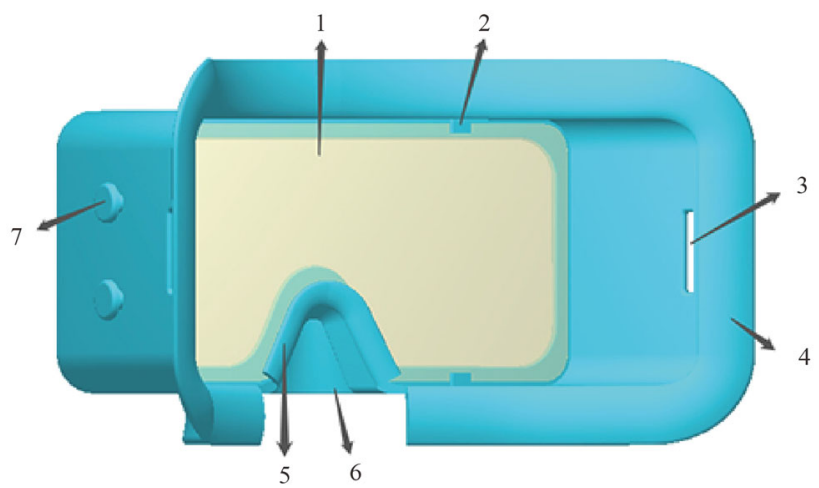

Fig. 1 Goggle model (1: lens; 2: slot; 3: elastic band holes; 4: outward flanging; 5: inward flanging; 6: nose cover; 7: ancho)

For satisfying requirement $\mathrm{D}$ in Table 1 , we designed two types of lenses: a single-lens and a double-lens (see Fig. 2). The single-lens has a wider field of vision, but the cutting process is more complex. The double-lens shape is square, which can simplify the lens cutting process. Four slots on the interior of the front panel (see "2" in Fig. 1) were added to reinforce the lenses.

For satisfying requirement $\mathrm{E}$ in Table 1, we avoided sharp edges and corners in the design process and designed the nose cover for the exposed part of the nose (see " 6 " in Fig. 1).

We designed six types of goggles: 3DP-S-L, 3DP-S-M, 3DP-S-S, 3DP-D-L, 3DP-D-M, and 3DP-D-S. In the labels, "3DP" indicates that the manufacturing method is based on 3D printing. Regarding the middle letter, " $S$ " represents "single-lens"; and "D" represents "double-lens". Regarding the last letter, "L" represents "large"; "M" represents "medium"; and "S" represents "small" (only for children). Details are presented in Fig. 2.

\section{Preparation of eye-face shield (3DP-S-M considered for example)}

This section describes the preparation process for the eyeface shield, including the production of the 3D-printed goggles, lens, and shield and the assembly process of the eye-face shield. For users to quickly understand the manufacturing method of the eye-face shield, the manufacturing details are disclosed in detail here. We are willing to 


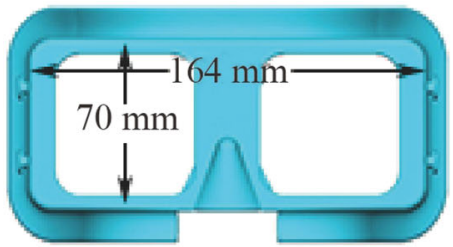

(a) 3DP-S-L

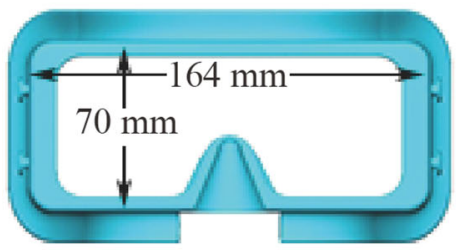

(d) 3DP-D-L

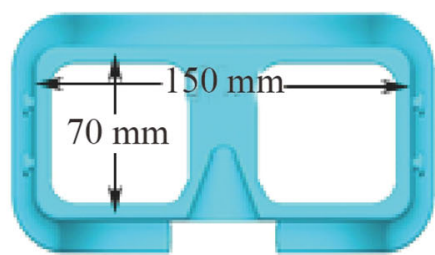

(b) 3DP-S-M

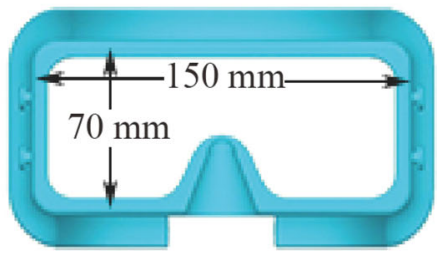

(e) 3DP-D-M

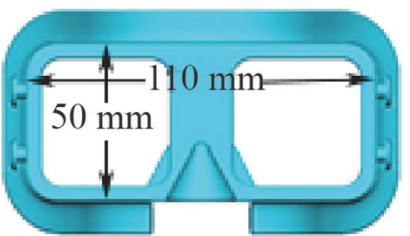

(c) $3 \mathrm{DP}-\mathrm{S}-\mathrm{S}$

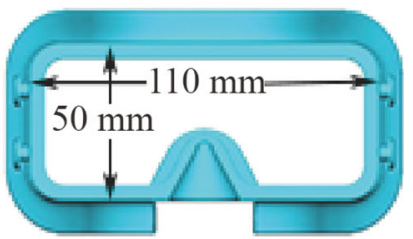

(f) 3DP-D-S

Fig. 2 Schematic representations of the six types of goggles

mobilize all possible social resources to help front-line medical personnel fight against the epidemic.

\subsection{D-printed goggles}

After printing the goggle samples, we tried them on and modified them repeatedly, and the final material and printing method were determined. This article recommends the use of the stereolithography (SLA) printing method. The material should be selected from medical, safe photosensitive resin, or thermoplastic polyurethane elastomer. After the goggle model is complete, the user should save it as STL files, which are then imported into the printing software for parameter setting before printing. Firstly, supports should be established for the model. The support setting style is shown in Fig. 3. The printing parameters are important, as they determine the quality of the sample. After many printing cycles, we obtained the optimal printing parameters, and the success rate was $100 \%$. When setting the printing parameters, one can refer to the main printing parameters presented in Table 3. After completing the printing-parameter setting, a print preview should be performed. Figure 4 shows the correct print preview of some layers. In the checking of the print preview, a different posture of the model and different parameter settings will make the preview image different. If one sets the parameters to be consistent with the parameters that we provided and is unsure whether the print preview is correct, one can refer to Fig. 4. Alternatively, he/she should consult a 3D-printing professional.

\subsection{Assembly process of eye-face shield}

The eye-face shield includes four parts: the face shield, lenses, goggles, and elastic band (see Fig. 5). The assembly of the eye-face shield can be divided into three steps. The first step is to apply glue to the lens and attach it to the goggles, during which the users should pay attention to the slots. The second step is to pass the end of the elastic band through the elastic-band holes and then fix it by stitching, gluing, knotting, and other methods. The third step is to press the cross hole on the face shield into the four anchors. The corresponding relationship is shown in Fig. 6. The assembled eye-face shield is shown in Fig. 7.
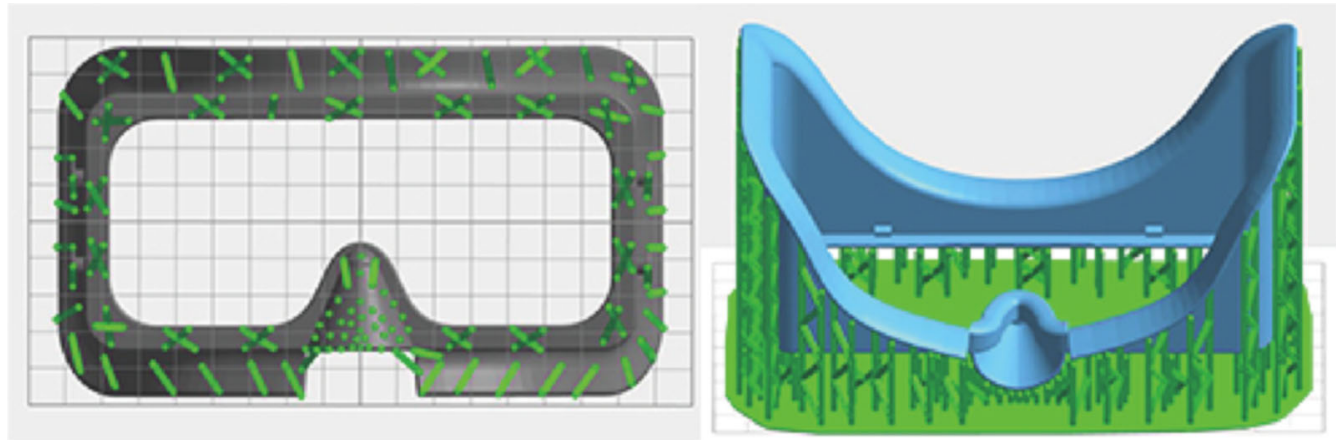

Fig. 3 Support settings 
Table 3 Main parameters of 3D printing

\begin{tabular}{llccc}
\hline Layer & Slice thickness/mm & Exposure time/s & Cooling time/s & LED power/W \\
\hline $0-3$ & 0.100 & 17.0 & 5 & 300 \\
$4-59$ & 0.100 & 8.0 & 5 & 300 \\
60 & 0.100 & 4.0 & 5 & 300 \\
\hline
\end{tabular}

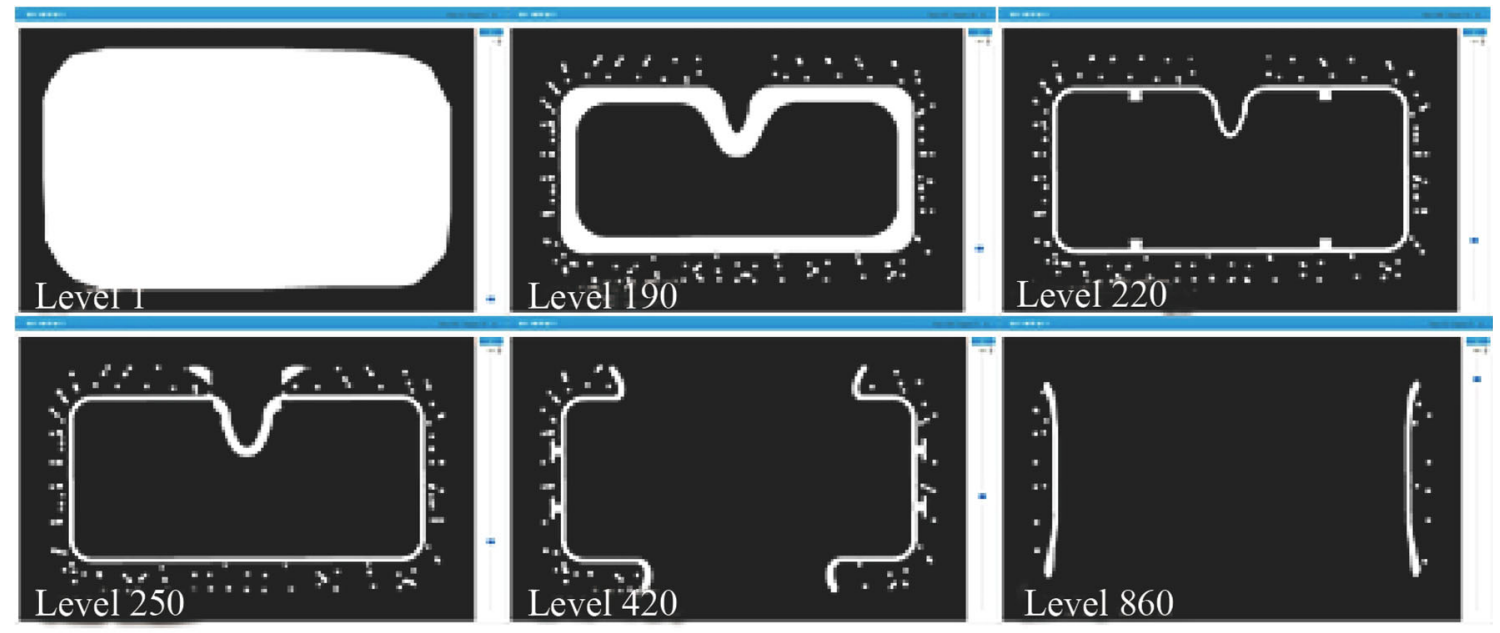

Fig. 4 Print preview of some layers

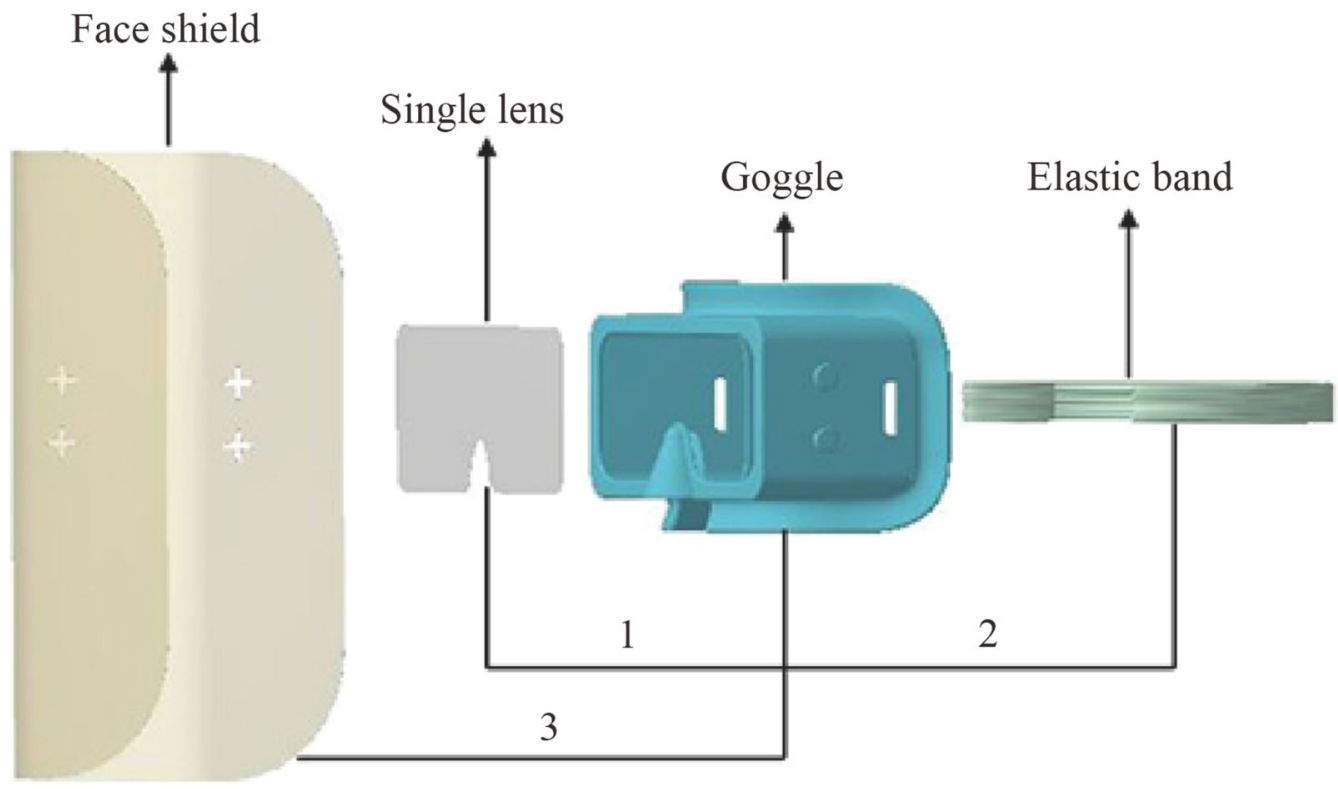

Fig. 5 Exploded view of eye-face shield

For face shield section, there are three sizes of the eyeface shield. For users to understand the preparation of the eye-face shield more accurately, pertinent documents are presented at the end of the article. Considering the different bending stiffnesses of transparent plastic sheets with different thicknesses, we recommend using a $0.5-1.0 \mathrm{~mm}$ transparent PVC plastic sheet after trial production. In lens section, in designing the eye-face shield, a single-lens and a double-lens were considered, for allowing individuals or small groups to fabricate the eye-face shield without limitations on the cutting process. The double-lens cutting process is easier, and the users simply need to cut a square 
transparent plastic sheet. In the single-lens eye-face shield, the middle baffle is removed, and the field of vision is larger. When the cutting process is not limited, it is recommended to use a single-lens eye-face shield. The lens files can be accessed via the link presented at the end of this article. The gaps of the lens slots are $1.5 \mathrm{~mm}$ wide, and the users must reserve the installation space. For the eyeface shield lenses, a transparent plastic sheet with a thickness of $0.5-1.0 \mathrm{~mm}$ is recommended.

\section{Discussion}

Regarding effectiveness, the eye-face shield has been used in many hospitals in Shanghai, China since February 5 2020. To date, more than 300 medical personnel have used the eye-face shield, none of whom were reported to be infected by COVID-19 (see Fig. 8). This result preliminarily confirms the effectiveness of the eye-face shield for

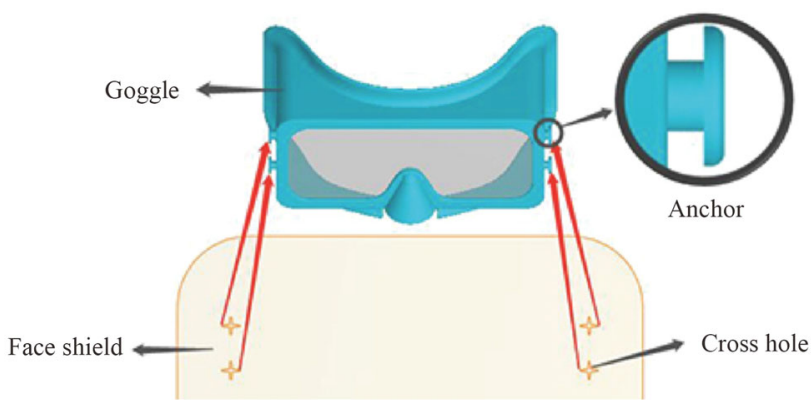

Fig. 6 Correspondence between cross holes and anchors

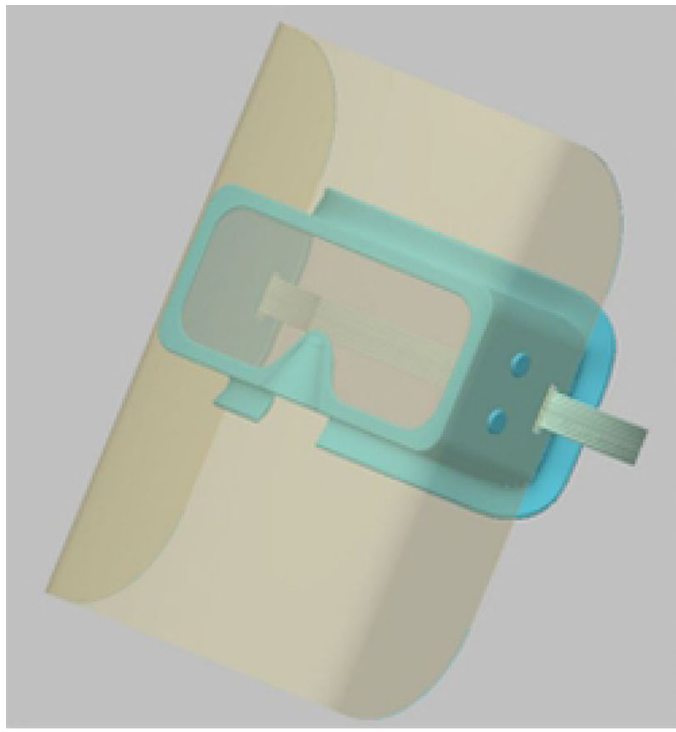

(a) protection. Regarding the issue of fogging, commercially available anti-fogging liquid, anti-fogging paste, or antifogging agents for swimming goggles can be considered. In the case of a shortage of the foregoing materials, antibacterial hand sanitizer, soap detergent, iodophor, or other substances can be smeared on the lens and surface to prevent fogging [7]. Regarding the manufacturing method, the applicability of 3D printing was discussed and validated in the design of the model, confirming that the best

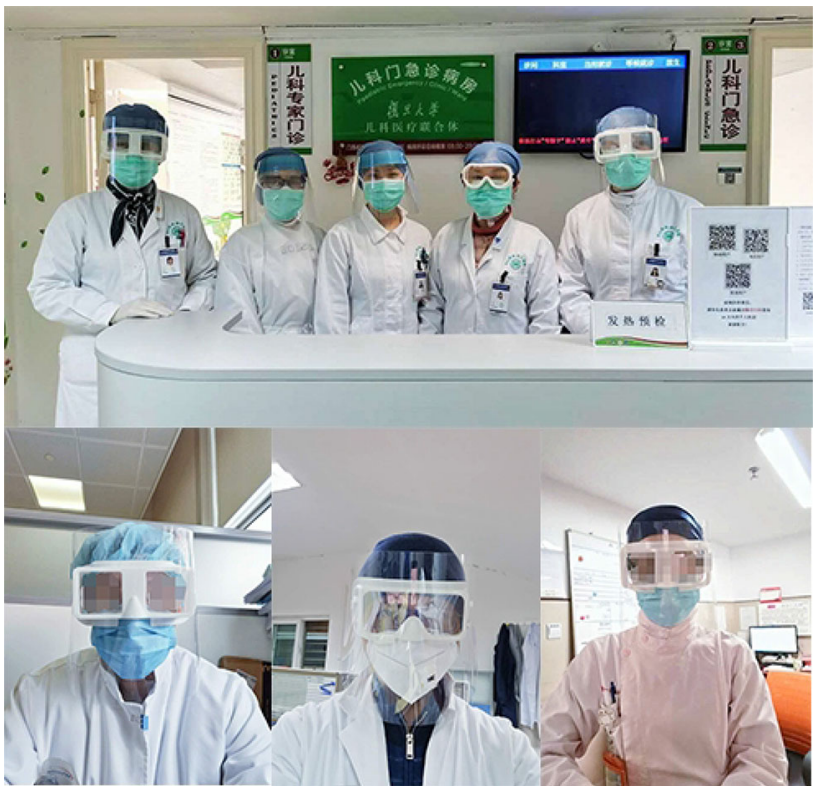

Fig. 8 Medical staffs wear our eye-face shields in working environment

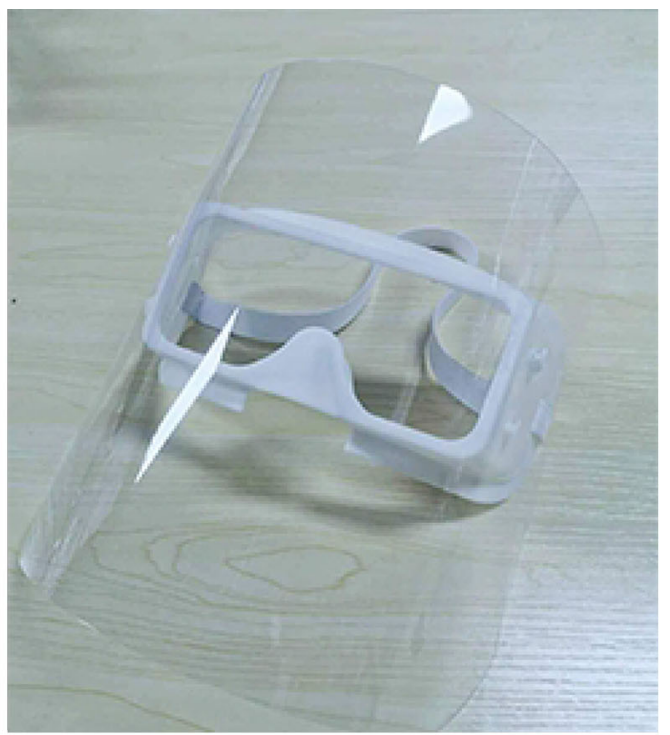

(b)

Fig. 7 Eye-face shield a effect picture and $\mathbf{b}$ physical picture of 3DP-S-M 
manufacturing method is 3D printing. At present, the daily output of face-eye shields with 3D printing in China can reach 2000 pieces [8]. Additionally, in China, companies have used $3 \mathrm{D}$ printing to produce samples for making molds. After the mold was obtained, face-eye shields were prepared using traditional manufacturing methods such as injection molding and compression molding. This not only provided more possibilities for the production of face-eye shields but also saved time and reduced costs. Of course, users are encouraged to try other production methods.

\section{Conclusions}

We proposed a new design and fabrication method for the rapid production of eye-face shields during the COVID-19 pandemic. The most significant parts of our proposition are the combined design of the eye-face shield and the 3D printing-based fabrication methods. With the proposed design, the entire product can be easily assembled and disassembled while maintaining a good anti-leakage effect against infectious droplets. Moreover, the proposed 3Dprinting technology allows the rapid preparation of the product, which is crucial in dealing with emergent infectious disease events such as COVID-19. The clinical effectiveness of our eye-face shield follows naturally from the all-in-one design and was preliminarily validated by the fact that more than 300 medical personnel in China have used our product since February 5 2020, none of whom were reported to be infected with COVID-19.

\section{Supporting material}

Digital models of our eye-face shields can be freely downloaded, used, or re-edited by anyone who needs them, for educational, research, or commercial purposes. Download link (Baidu cloud disk): https://pan.baidu.com/s/ 13C5gchZnBGtcJsn_p6i2SA Extraction code 349c

\section{References}

1. WHO (2020) Report of the WHO-China joint mission on coronavirus disease 2019 (COVID-2019).http://www.who.int/ docs/default-source/coronaviruse/who-china-joint-mission-oncovid-19-final-report.pdf. Accessed 4 Mar 2020

2. Lu CW, Liu XF, Jia ZF (2020) 2019-nCoV transmission through the ocular surface must not be ignored. Lancet (London, England) 395(10224):e39
3. Wang X, Pan Z, Cheng Z (2020) Association between 2019-nCoV transmission and N95 respirator use. J Hosp Infect 105(1):104-105

4. National Health Commission of the People's Republic of China (2020) Guidance on the scope of use of common medical protective equipment in the prevention and control of pneumonia caused by new coronavirus infection (for trial implementation). http://www.nhc.gov.cn/yzygj/s7659/202001/e71c5de925a64eafbe1 ce790debab5c6.shtml

5. Tino R, Moore R, Antoline S et al (2020) COVID-19 and the role of 3D printing in medicine. 3D Print Med 6:11. https://doi.org/10. 1186/s41205-020-00064-7

6. Neijhoft J, Viertmann T, Meier S et al (2020) Manufacturing and supply of face shields in hospital operation in case of unclear and confirmed COVID-19 infection status of patients. Eur J Trauma Emerg Surg 46:743-745

7. Huang XQ, Qu J, Chen YY et al (2020) Correct choice of goggles and anti-fog guidance during the COVID-19 epidemic period. Chin J Optom Ophthalmol Vis Sci 22(00):E002-E002. https://doi. org/10.3760/cma.j.cn115909-20200301-00054

8. VANXSANWEI (2020) [3D Digital Application] 3D printed medical goggles help the epidemic. http://www.wanxiang3d.com/ h-nd-148.html

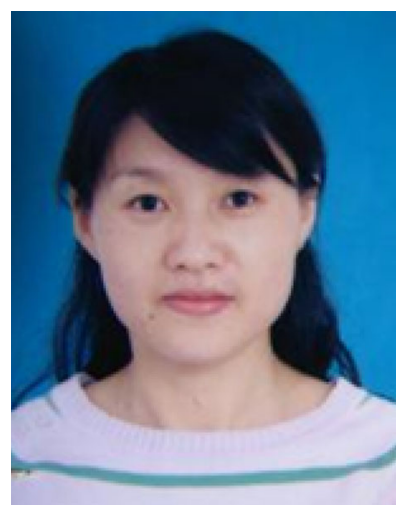

Xiu-ling Huang received her $\mathrm{Ph} . \mathrm{D}$. degree from Jiangnan University, China. She is an associated professor at the School of Mechatronic Engineering and Automation, Shanghai University. She is currently the deputy director of the Intelligent Basics Parts Research Center of Shanghai University and a member of the 3D Printing Medical Device Special Committee. Research interests: wearable rehabilitation aids, intelligent orthopedic implants, advanced medical device technology.

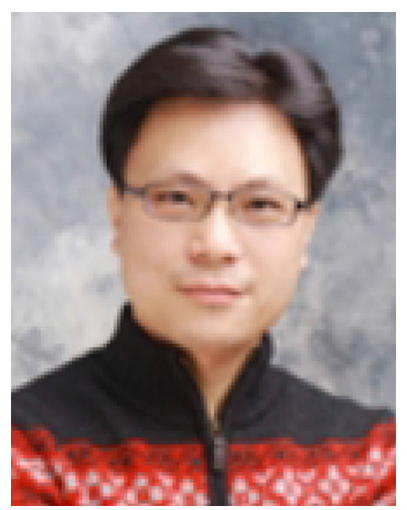

Zi-kai Hua is the director of the Intelligent Mechanical Elements Research Center (Shanghai University), director of the Orthotek Laboratory (ISO17025 accredited), and vice dean of Shanghai Xuhui Center Hospital (appoint on secondment, 2016). Dr. Hua is invited as a visiting professor at the JointsInSilico Research Center at Imperial College London, KTP fellow of University of Technology Sydney, etc. Prof. Hua developed long-term Medical \& Engineering Interdisciplinary with hospitals and studies the engineering problem in Translational Medicine at the aspects of rehabilitation engineering, surgical implants, etc. 